دور كريات الدم الحمر في نمو الاطوار المتغذية للأميبا الحالّة للنسج في الزجاج (Entamoeba histolytica)

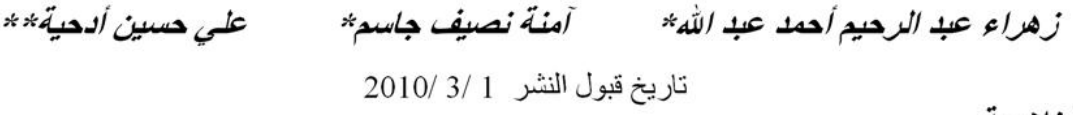

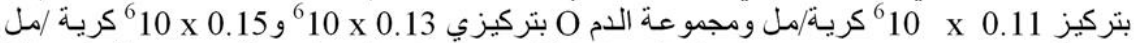

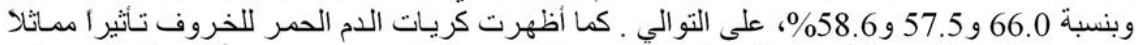

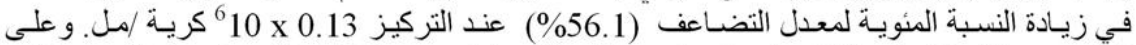

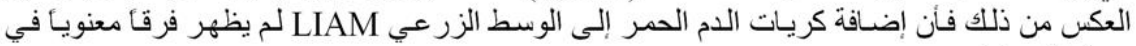
معدل التضاعف دن تل

كلمات مفتاحية: أستنبات ، الاميبا الحالة للنستج ، كريات الدم الحمر

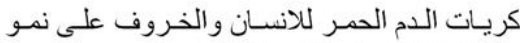
ونثاط الطفيلي في الاوساط الزر عية.

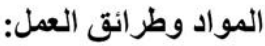
جمع وعزل الطفيلي من عينة البراز البراز جمعت عينات البراز من أنثخ البراص باز بالغين

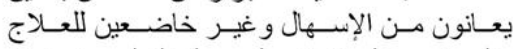

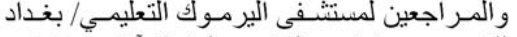

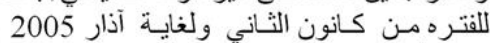
وتم التأكد من خمجهم بطفيلي الأمييا الحالتة للنستج

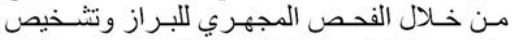

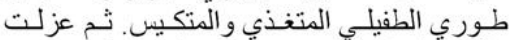

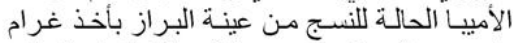

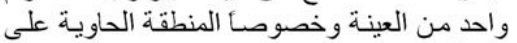

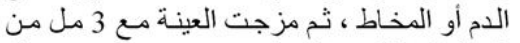

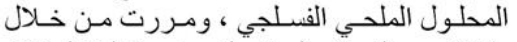

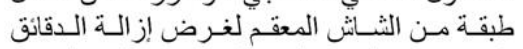

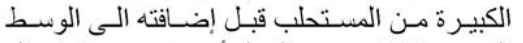

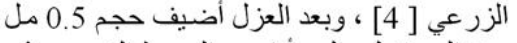

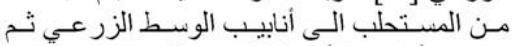

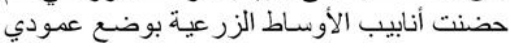

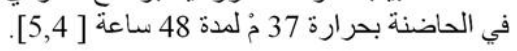

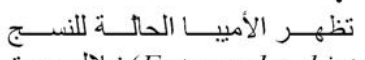

خـال دورة حياتها (Entamoeba histolytica)

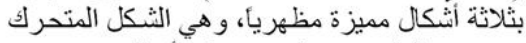

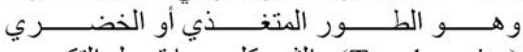

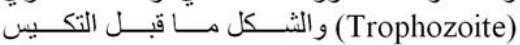
(Precyst)

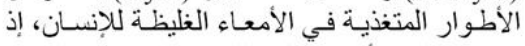

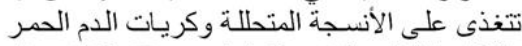

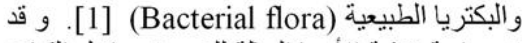
تم دراسة تغذية الأميبا الحالة للنسج في داخل الكائن

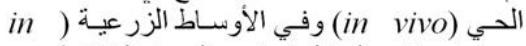

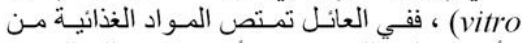

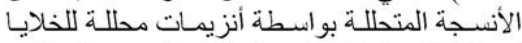

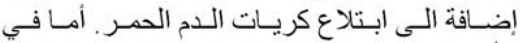

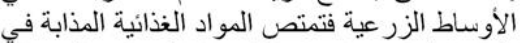

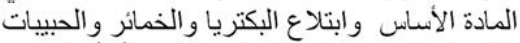

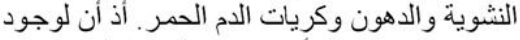

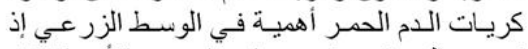

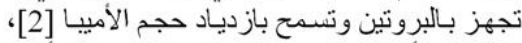

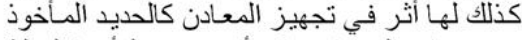

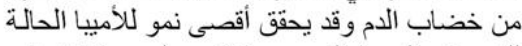

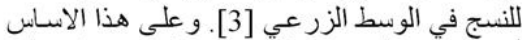

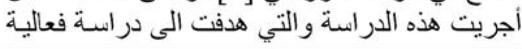

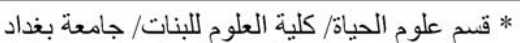
*** * قحدة الأبحاث البايولوجية للمناطق الحارة /كلية العلوم جامعة بغداد 


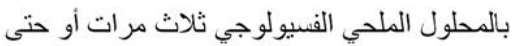

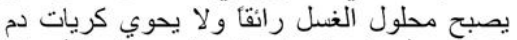
متحللة، أضيفت كريات الدم الحمر ألى الأوساط

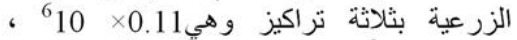
0.13

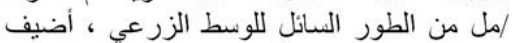

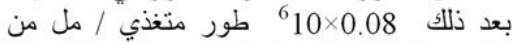

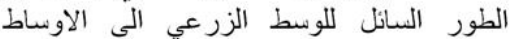

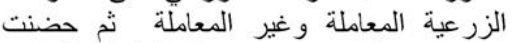

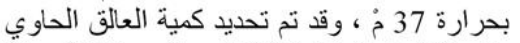
على الاطو ار المتغذية للطفيلي والمضافة لكيدة للاوساط

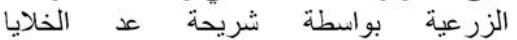
[6] Haemocytometer

قياس فعالية المعاملة تم قياس فعالية المعاملة بأستخدام المعادلة الاتية وحسب ماجاء في [7]

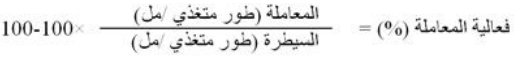

Statistical Analysis التحليل الإحصائي حلت النتائج باستخدام اختبار أقل فرق معنوي (LSD) Least significant difference وكذلك استعمل اختبار دنكن المتعدد المدى Duncan Multiple Range Test البرنامج الاحصائي الجاهز (SPSS )

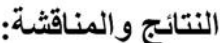

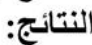

استتد طيلة مدة البحث على سـلالة أمييية

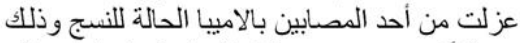

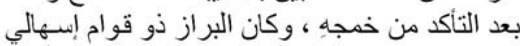

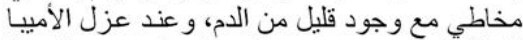

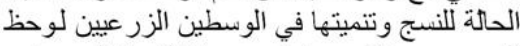

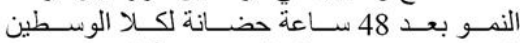

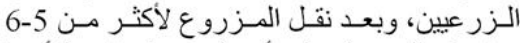

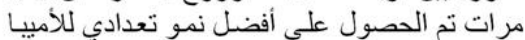

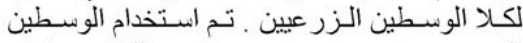
الزر عيين (LEM) و (LIAM) و واللذان يمتاز التونين

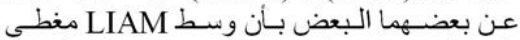
بالمصل بينما وسط LEM لا يحتوي على البعل المصل

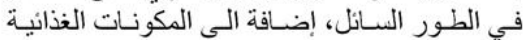

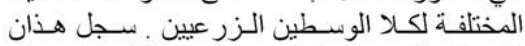

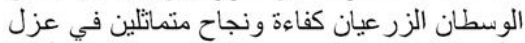
الاميبا من البراز وكانت نسبة نمبة نمو ونثـاط الأمييا

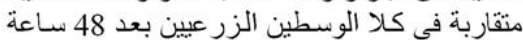

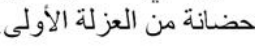

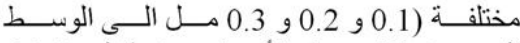
الزرعي) وكانت هذه الأحجام مسـاوية الى 0.11 (0.11
Culture تحضير الأوساط الزرعية

حضرت نوعين من الأوساط الزرعية

\section{Media}

من نوع Xenic culture media لتنمية الاميبا الحالة للنسج، و هذه الأوساط ذات بيئة ثنائية الطور

(Diphasic media)

Locke- egg (LE) ألوسط الزرعي (DE)

medium

(LE)

حضر الوسط الزرعي والذي يتكون من

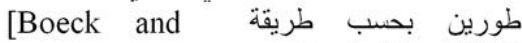
Drobohlav, 1925]

1. الطور الصلب : أن المكون الأساسي لهذا

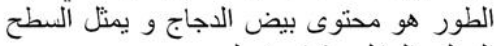

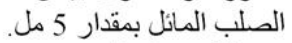

2. الطور السائل : محتوى هذار مل الطور هو

محلول لوكس (Locke's solution) و لطون يمنل

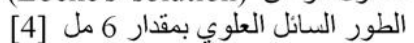

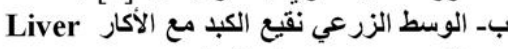
infusion agar medium

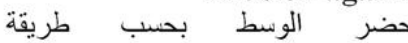

ويتكون (Cleveland and Collier,1930)

ايضأ من طورين : 1. الطور الصلب : أن المكون الأساسي لهذا

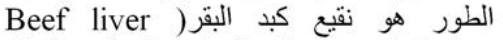
(infusion بمقدار 5 مل المبانر

2. الطور السائل: يتألف هذا الطور من دارئ

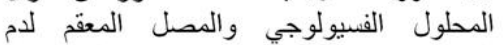
الخروف ، إذ مزجا بنسبة 1:5 الفيول ، والذي بمثل

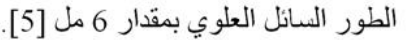
Antibiotics المضادات الحيوية الطبة

أضيف كل من Streptomycin Procaine بمقدار 2 ملغم/مل من Sulphate و بمقدار 1000وحدة دولية/مل و Nystatin بمقدار 2 ملغم/مل اللى الطور السائل اللوسط الزر عي [4 ب

$$
\text { وتهيئة عينات دم الإنسان والخروف وتوصيفها }
$$

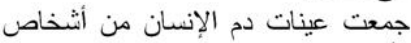

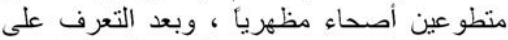

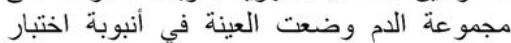

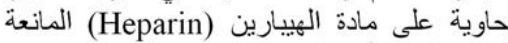

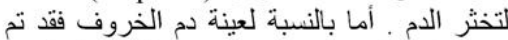

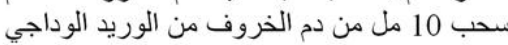

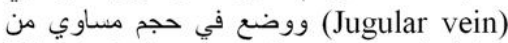
محلول السفير ـ وبعد التخلص من البلازما وطبقيقة

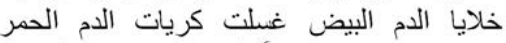

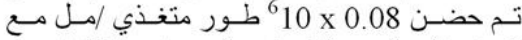
كريات الدم الحمر للانسان والخروف بثثّلاث احجـام 
كمـا أظهرت النتائج عدم وجـود فـروق

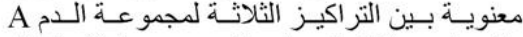
والسيطرة و لكلا الوسطين الزر عيين وكذللك الحال

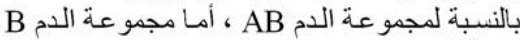

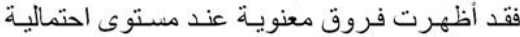

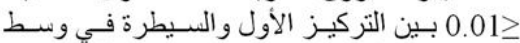

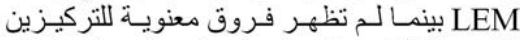

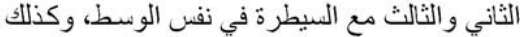

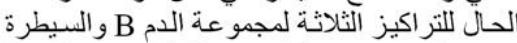
في وسط LIAM، أما مجمو عة الدم

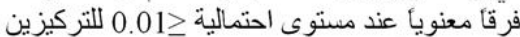

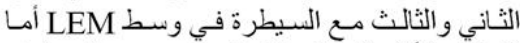
التركيز الأول فلم يلاحظ فرق معنوي مع السيطرة،

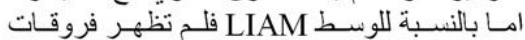

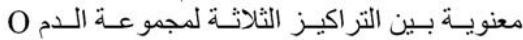

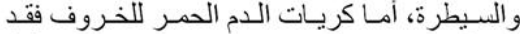

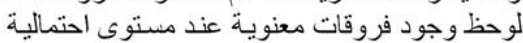

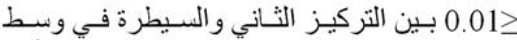

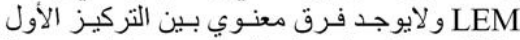

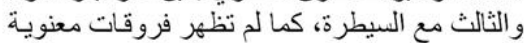

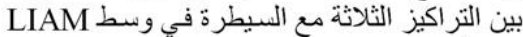

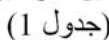

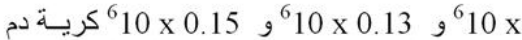

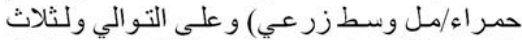

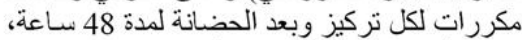
لوحظ أن التركيز الأول كان أفضل من التركيزين

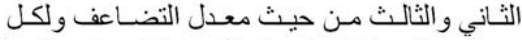

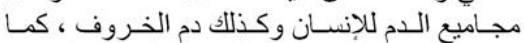

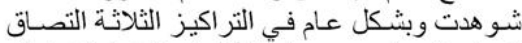
كريات الدم الحمر بسطح الأطوار المتغذية ( شكل التران

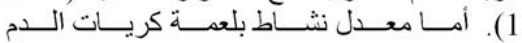

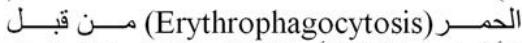
الأطوار المتغذية للامييا فكانت متباينة وكذلك الحال

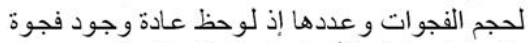

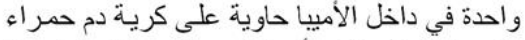

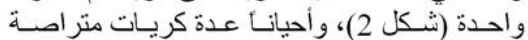

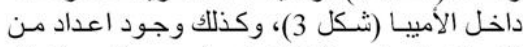

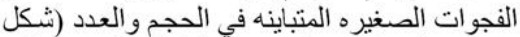

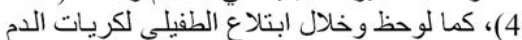

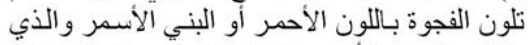

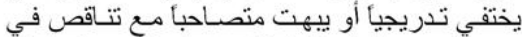

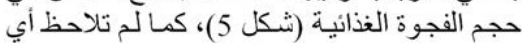

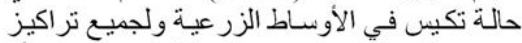

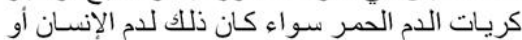

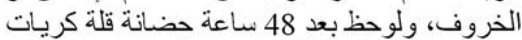
الدم الحمر للتركيز الأول والثاني أما التركيز الثالثة

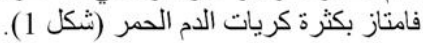

جدول (1) : تأثير كريات الدم الحمر للإنسان والخروف على نمو طفيلي الأميبا الحالة للنَّجُ و النامية

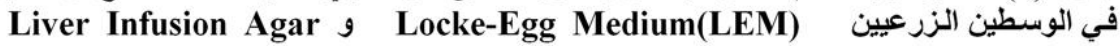
. Medium(LIAM)

\begin{tabular}{|c|c|c|c|c|c|c|c|}
\hline \multirow{2}{*}{$\begin{array}{c}\text { الاحتمائية * } \\
\geq\end{array}$} & \multicolumn{2}{|c|}{ فُعائية المعاملة (\%) في } & \multicolumn{2}{|c|}{ 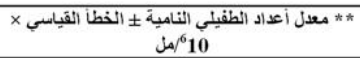 } & \multirow{2}{*}{ 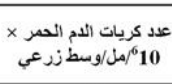 } & \multirow{2}{*}{\multicolumn{2}{|c|}{ المجاميع }} \\
\hline & LIAM & LEM & LIAM & LEM & & & \\
\hline 0.001 & & & $0.085 \pm 0.386$ & $\begin{array}{l}\quad 0.084 \pm 0.905 \\
\end{array}$ & 0.00 & \multicolumn{2}{|c|}{ السيطرة } \\
\hline 0.001 & $4.4+$ & $18.8+$ & $0.026 \pm 0.403$ & $\begin{array}{ll}\mathrm{i} & 0.014 \pm 1.076 \\
\end{array}$ & 0.11 & \multirow{3}{*}{ A } & \multirow{3}{*}{$\frac{3}{3}$} \\
\hline 0.01 & $37.0-$ & $3.2-$ & $0.028 \pm 0.243$ & $\rightarrow 0.115 \pm 0.876$ & 0.13 & & \\
\hline 0.001 & 27.4 & $5.6+$ & $0.01 \pm 0.280$ & $\begin{array}{ll} & 0.080 \pm 0.956\end{array}$ & 0.15 & & \\
\hline 0.01 & $37.0-$ & $66.0+$ & $0.035 \pm 0.243$ & $\begin{array}{ll} & 0.17 \pm 1.503\end{array}$ & 0.11 & \multirow{3}{*}{ B } & \\
\hline 0.01 & $32.6-$ & $40.6+$ & $0.03 \pm 0.260$ & $0.184 \pm 1.273$ & 0.13 & & \\
\hline 0.001 & $58.5-$ & $36.2+$ & $\rightarrow \square 0.023 \pm 0.160$ & $0.066 \pm 1.233$ & 0.15 & & \\
\hline 0.01 & $76.9+$ & $33.2+$ & $0.063 \pm 0.683$ & $0.070 \pm 1.206$ & 0.11 & \multirow{3}{*}{ AB } & \\
\hline 0.001 & $20.7-$ & $29.2+$ & $0.038 \pm 0.306$ & $0.015 \pm 1.170$ & 0.13 & & 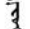 \\
\hline 0.001 & $52.5-$ & $29.9+$ & $\rightarrow-0.033 \pm 0.183$ & $0.034 \pm 1.176$ & 0.15 & & . \\
\hline 0.001 & $55.1-$ & $15.5+$ & $\mp 0.028 \pm 0.173$ & $0.026 \pm 1.046$ & 0.11 & \multirow{3}{*}{ o } & \\
\hline 0.001 & $45.5-$ & $57.5+$ & $0.011 \pm 0.210$ & $0.078 \pm 1.426$ & 0.13 & & 3 \\
\hline 0.001 & $8.8+$ & $58.6+$ & $0.087 \pm 0.420$ & $\triangle 0.003 \pm 1.436$ & 0.15 & & \\
\hline 0.001 & $44.0-$ & $6.0+$ & $0.020 \pm 0.216$ & $0.049 \pm 0.960$ & 0.11 & \multirow{3}{*}{\multicolumn{2}{|c|}{ كريات الثم الحمز }} \\
\hline 0.001 & $64.7-$ & $56.1+$ & $\rightarrow 0.042 \pm 0.136$ & $0.131 \pm 1.413$ & 0.13 & & \\
\hline 0.001 & $40.4-$ & $3.0+$ & $0.034 \pm 0.230$ & $0.038 \pm 0.933$ & 0.15 & & \\
\hline
\end{tabular}

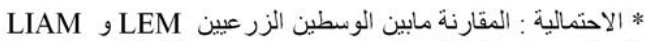

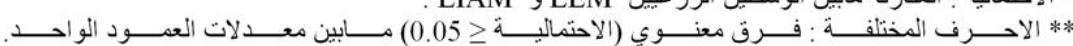



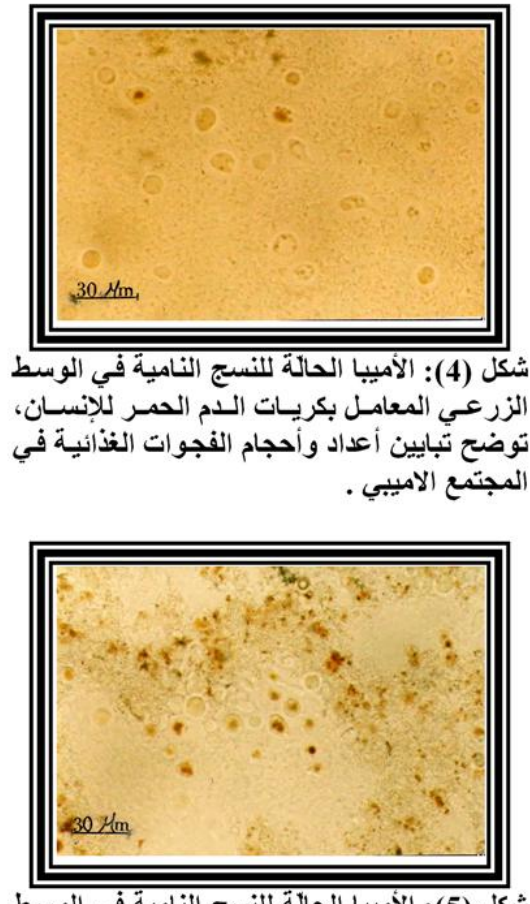

شكل (5): الأميبا الحالة للنسج النامية في الوسط الزيط

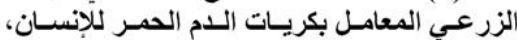

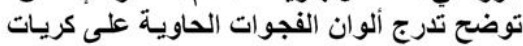
الام الحمر بحسب مراحل الهضم الداخل خلوي.

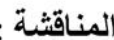

بينت النتائج نجاح عزل الأمييا من البراز

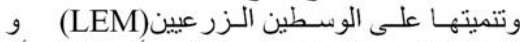

(LIAM)

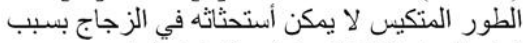
توفر المواد الغذائية وغياب التحفيز البيئي. اما بالنسبة للوسطين الزئب الزيز عيين فقد أظهر

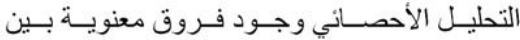

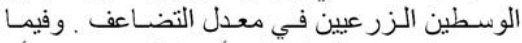

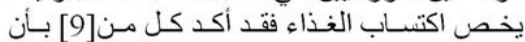

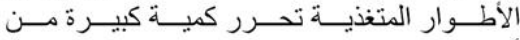

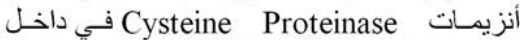

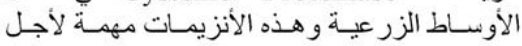

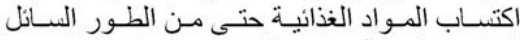

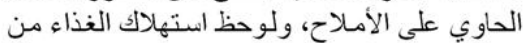

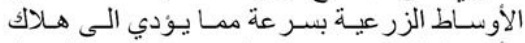

الأمييـا لذلك تلتم الادامـة كل 48-72 سـاعة لتبديل

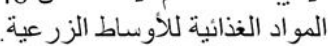

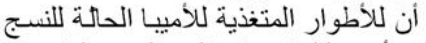

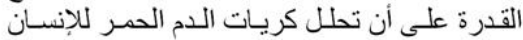

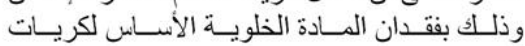

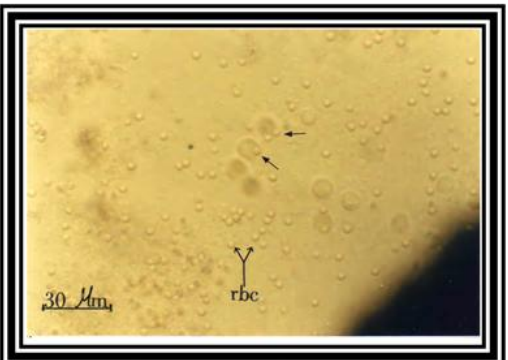

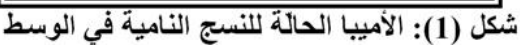

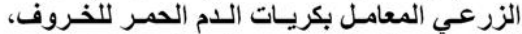

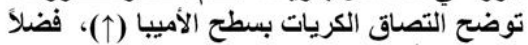

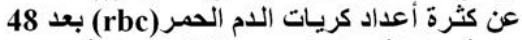
ساعة حضانة عند التركيز الثالث للمعاملة.

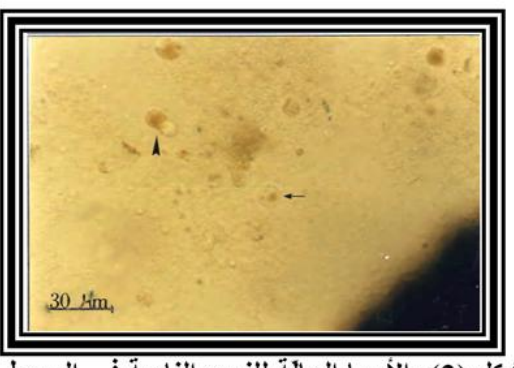

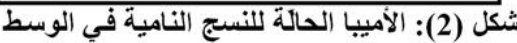

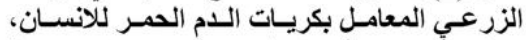

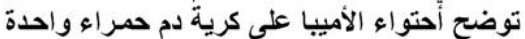

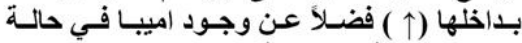
انقسام وحاوية على كرية دم حمر اءو ( ).

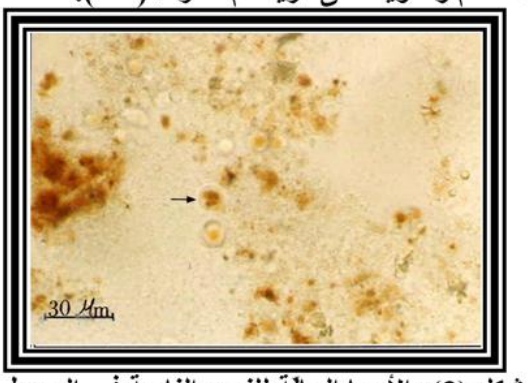

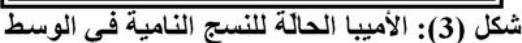

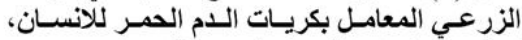

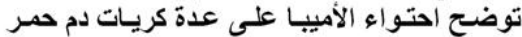
متر اصة ( ) ). 


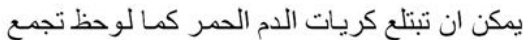

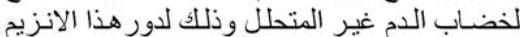

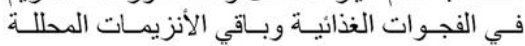

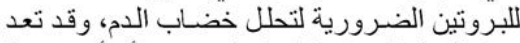

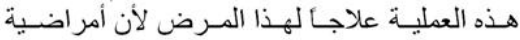

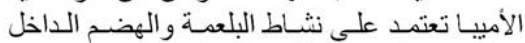
والخـار جلو تعلوي وكذلك تحلل البروتين و باستخدام الإثبطات لهذا الانزيم قد يؤدي ذلإ الك الى اعاقة هجوم

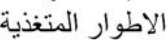

إذن يتطلب تنمية الأمييا الحالة للنسج في

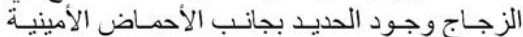

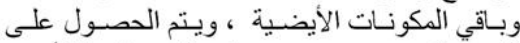

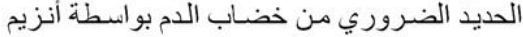
Hemoglobinase المبتلعة [13]. وذكر [14] بـأن الأطوار المتغذية

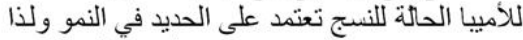

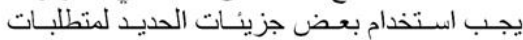

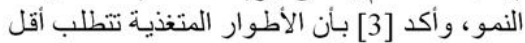

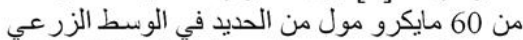

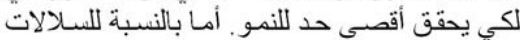

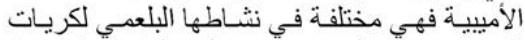

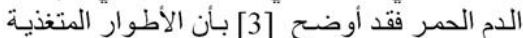

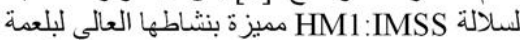

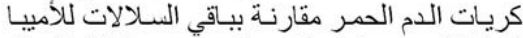

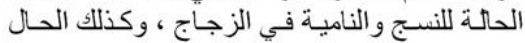

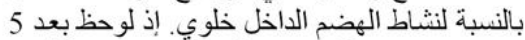

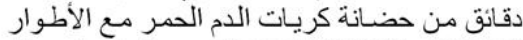

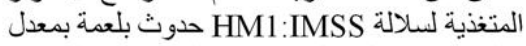

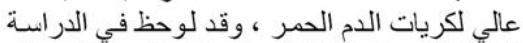

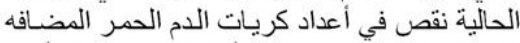

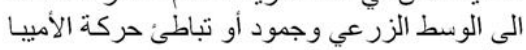

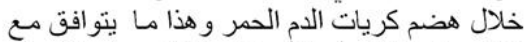

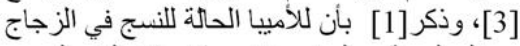

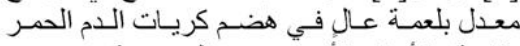

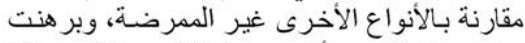

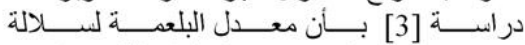
HM1:IMSS حمر اء لكل طور متغذي خلال خمس دقائق، و ولى بلى الر غم من ذلك فـأن مجتّمع (Population) الأمييا

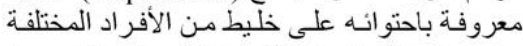

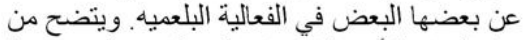

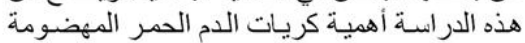

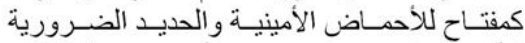

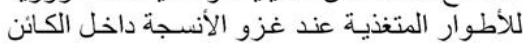

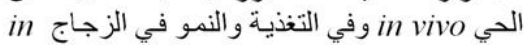

vitro
الدم)(Erythrocyte cellular matrix) وتحلل الغثاء البلازمي بحسب مـا شـاهده[3] ويستمر مـع

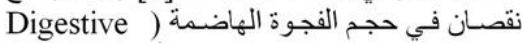

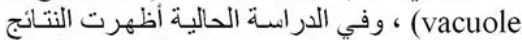

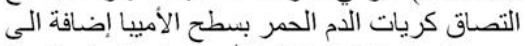

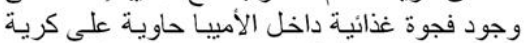

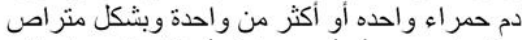

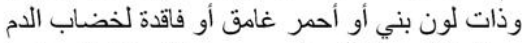

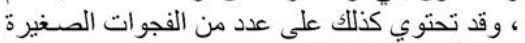

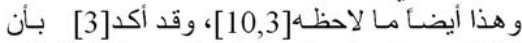

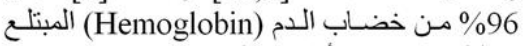

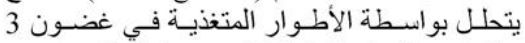

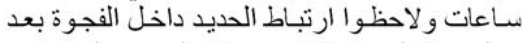

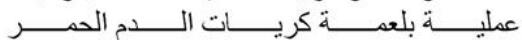
إذ شو هد ذلك بو اسطة الحة (Erythrophagocytosis) X-ray spectroscopy يتحدد نثاط الاطوار المتغذية بمجموعة

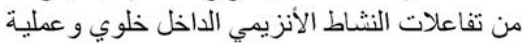

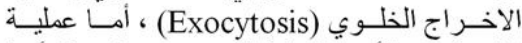

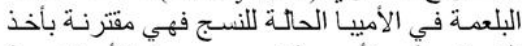

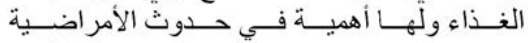

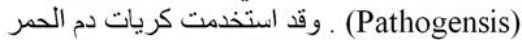

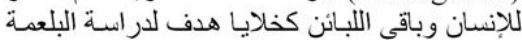

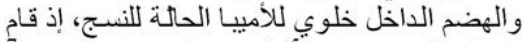

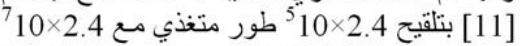

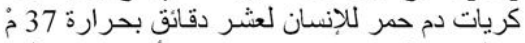

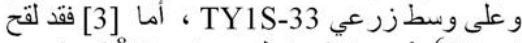

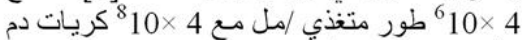

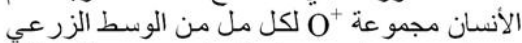

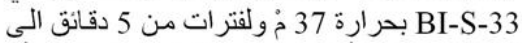

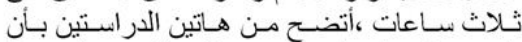

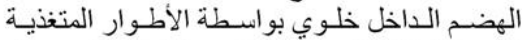

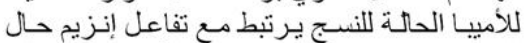

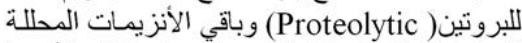

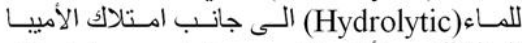
الحالة للنستج لأنزيمات Cysteine proteinases،

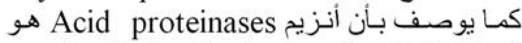

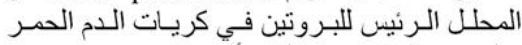

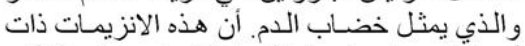
أهمية كبيرة في فو عة الأمييا إذ لها دور في الأني التأثير

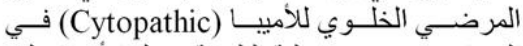
الزجاج [3] ، فقي عملية البلعمة تعمل الأميبـ على لـي

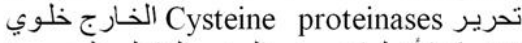

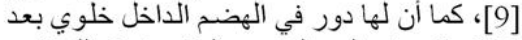

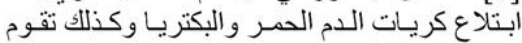

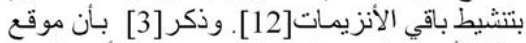

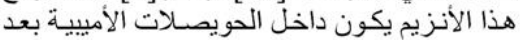

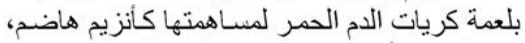

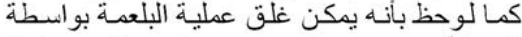

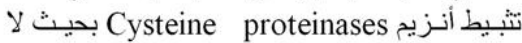


9. Que, X.and Reed, S.L. 2000. Cystine proteinases and the pathogenesis of amebiasis. Clin. Microbiol. Rev., 13:196-206.

10. Mora-Galindo, J. and AnayaVelazquez, F. 1993. Intracellular digestion of human erythrocytes by Entamoeba histolytica: a kinetic study in vitro . Arch. Med. Res., 24: 347-351. [Abstract]

11. Labruyere, E.; Zimmer, C.; Galy, V.; Olivo-Marin, J.C. and Guillen, N. 2003. EhPAK, a member of the P21-activated kinase family, is involved in the control of Entamoeba histolytica migration and phagocytosis. J. Cell Sci., 116:61-71.

12. Stanley, S. L. and Reed, S.L. 2001. Microbes and microbial toxins: Paradigms for microbial-mucosal interactions VI. Entamoeba histolytica : parasite- host interactions. Am. J. Physiol Gastrointest liver Physiol., 280:G1049-G1054.

13. Espinosa-Cantellano, M. and Martinez-Palomo, A. 2000. Pathogenesis of intestinal amebiasis : From molecules to disease. Clin. Microbiol. Rev., 15:318-331.

14. Reyes-Lopez, M. ; Serrano-Luna, J.J.; Negrete-Abascal, E.; Sicairos, N.; Guerrero-Barrera, A.L. and dela-Garza, M. 2001. Entamoeba histolytica : transferrin binding proteins. Exp. Parasitol., 99:132140. [Abstract].

15. Dagci, H.; Balcioglu, C.; Ertabaklar, H.; Kurt, O. and Atambay, M. 2003. Effectiveness of peptone-yeast extract (P-Y) medium in the cultivation and isolation of Entamoeba histolytica / Entamoeba dispar in Turkish patients. Diagn. Microbiol. Infect. Dis., 45:127-130.

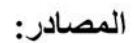

1. Tanyuksel, M. and Petri, W.A. 2003. Laboratory Diagnosis of Amoebiasis. Clin. Microbiol. Rev., 16:713-729.

2. Rifaat, M.A. and Morsy, T.A. 1967. Manual of Medical Parasitology, $2^{\text {nd }}$ ed. Dar Memphis, Cairo: pp 193-197.

3. Mora-Galindo, J.; AnayaVelazquez, F. ; Ramirez-Romo, S. and Gonzalez-Robles, A. 2004. Entamoeba histolytica : correlation of assessment methods to measure erythrocyte digestion, and effect of cysteine proteinases inhibitors in HM-1: IMSS and HK-9:NIH strains. Exp. Parasitol., 108:89100.

4. Clark, C.G. and Diamond, L.S. 2002. Methods for cultivation of luminal parasitic protists of clinical importance. Clin. Microbiol. Rev., 15:329-341.

5. Taylor, A.E.R. and Baker, J.R. 1968. The cultivation of parasites in vitro. Black Well Science Publ., Oxford .pp.120-144.

6. Brousseau, P.; Payette, Y.; Tryphonas, H. Blakley, B.; Boermans, H.; Flipo, D. and Fournier, M 1999. Manual of immunological methods. CRC Press LIC, United State of America, Florida.pp7-135.

7. Lwin, K.M. and Oo, M. 2004. In vitro anteamoebicidial activity of "Dysenzi" on Entamoeba histolytica in cultures. FAME Pharmaceuticals Co., Ltd. Internet:http://Famepharma.com.

8. Barron-Gonzalez, M.P.; VillarrealTrevino, L.; Verduzco- Martinez, J.A.; Mata-Cardenas, B.D. and Morales-Vallarta, M.R. 2005. Entamoeba invadens: invitro axenic encystations with a serum substitute. Exp. Parasitol., 110:318-321. 


\title{
The role of some types of erythrocytes on the growth of Entamoeba histolytica trophozoite in vitro
}

\author{
Zahra'a Abdul-Raheem Ahmed* \\ Ali H. Ad'hiah** \\ Amna N. Jasim* \\ *Department of Biology, College of Science for Women, University of Baghdad \\ ** Tropical-Biological Research Unit, College of Science, University of Baghdad.
}

\begin{abstract}
:
The parasite E.histolytica was first isolated from a stool sample, and then cultivated and maintained in vitro using Locke-egg medium (LEM) and Liver infusion agar medium (LIAM) . Then, the effect of some types of erythrocytes (human and sheep), on the growth and activity of the parasite in the two culture media was investigated.

The parasite was able to ingest and lysis erythrocytes of human and sheep that were supplemented to the culture media and such manipulation was able to augment the reproduction rate of the cultivated $E$. histolytica, however, such consequence was media- and concentration-dependent. The reproduction rate was significantly increased (66.0, 57.5 and $58.6 \%$, respectively) in LEM medium containing human erythrocytes types $\mathrm{B}$ at $0.11 \times 10^{6}$ cells $/ \mathrm{ml}$ and $\mathrm{O}$ at $0.13 \times 10^{6}$ and $0.15 \times 10^{6}$ cells $/ \mathrm{ml}$. The sheep erythrocytes showed a similar enhancement $(56.1 \%)$ at a concentration of $0.13 \times 10^{6} \mathrm{cells} / \mathrm{ml}$. In contrast, adding erythrocytes to LIAM medium did not enhance the reproduction rate of the parasite significantly.
\end{abstract}

